\title{
Patrones de actividad de murciélagos filostómidos en un bosque
} seco en Honduras

\section{Activity patterns of phyllostomid bats in a dry forest in Honduras}

\author{
Manfredo A. Turcios-Casco ${ }^{*}{ }^{*}$, Hefer D. Ávila-Palma ${ }^{1}$, José A. Soler Orellana ${ }^{1}$, Eduardo J. Ordoñez Trejo ${ }^{1}$, y Diego I. Ordoñez Mazier ${ }^{1}$ \\ ${ }^{1}$ Asociación para la Sostenibilidad e Investigación Científica en Honduras (ASICH). Barrio La Granja, entre 28 y 29 calle, C. P. 504. \\ Comayagüela M.D.C. Francisco Morazán, Honduras. E-mail: manturcios@gmail.com (MAT-C), hefer.avila77@gmail.com (HDA-P), \\ biacuya00@yahoo.com (JASO), edu.ordnz03@gmail.com (EJOT), diego.ivano.mazier@gmail.com (DIOM). \\ *Corresponding author
}

\begin{abstract}
En Honduras, los estudios sobre murciélagos son escasos y se desconoce cómo la actividad de los murciélagos filostómidos es influenciada por factores ambientales. Describimos patrones de actividad de especies de murciélagos filostómidos en Ciudad Universitaria, Francisco Morazán (centro de Honduras). Realizamos 21 jornadas y capturamos murciélagos con 2 redes de niebla del 26 de febrero de 2015 al 29 de abril de 2016. En total, se registraron 114 individuos en 1,428.24 horas/red/metro. Con base en el análisis ANOVA, los murciélagos filostómidos se encontraron entre $58 \%$ y $93.5 \%$ de humedad relativa del aire, y entre $17.4{ }^{\circ} \mathrm{C}$ y $22.8^{\circ} \mathrm{C}$ de temperatura del aire. Nuestros resultados indican diferencias interespecíficas en la actividad de los murciélagos filostómidos en el área de estudio. Este es el primer intento en describir sus patrones de actividad en un bosque seco en Honduras.
\end{abstract}

Palabras clave: Ecología; factores ambientales; Honduras; patrones de actividad.

In Honduras, studies about bats are scarce, and it is unknown how the activity of phyllostomid bats is influenced by environmental factors. We describe activity patterns of phyllostomid bat species in Ciudad Universitaria, Francisco Morazán (central Honduras). We did 21 surveys and captured bats with 2 mist-nets from 26 February 2015 to 29 April 2016. Overall, 114 individuals were recorded in 1,428.24 net hours/meter. Based on ANOVA analysis, phyllostomid bats were found between $58 \%$ and $93.5 \%$ of the relative humidity of the air, and between $17.4{ }^{\circ} \mathrm{C}$ and $22.8^{\circ} \mathrm{C}$ of the temperature of the air. Our results indicate interspecific variations in activity of phyllostomid species in the study area. This is the first attempt to describe their activity patterns in a dry forest in Honduras.

Key words: Activity patterns; ecology; environmental factors; Honduras.

(c) 2021 Asociación Mexicana de Mastozoología, www.mastozoologiamexicana.org

La familia Phyllostomidae se caracteriza por la presencia de una hoja nasal, a excepción de algunas especies (por ejemplo, Centurio senex, Desmodus rotundus, Diphylla ecaudata, Diaemus youngii), la cual puede estar reducida o ser rudimentaria (Arita 1990). La hoja nasal es una estructura que les permite emitir y dirigir ultrasonidos para orientarse en el vuelo, encontrar su alimento y realizar vocalizaciones de carácter social (Galindo-González 1998; Orozco-Lugo et al. 2013). Además, pueden aprovechar diversos recursos alimenticios que les permiten participar en procesos ecológicos que incluyen dispersión y establecimiento de semillas, polinización y disminución de poblaciones de insectos (Neuweiler 1989; Arita y Fenton 1997; Kalko y Condon 1998; Vargas-Espinoza et al. 2008; Novoa et al. 2011; Cirranello et al. 2016). Ciertos factores como la disponibilidad de recursos alimentarios o variables climáticas pueden influenciar directamente su actividad, la cual se ve reflejada en la condición física, dieta y en los patrones de actividad (Estrada-Villegas et al. 2007; Muñoz-Romo y Herrera 2010; Novoa et al. 2011; García-García y Santos-Moreno 2014).

Si bien Amorim et al. (2012) mencionan que la temperatura y la humedad relativa del aire están relacionadas con la actividad de los murciélagos, en donde la actividad incrementa con altas temperaturas, no se conoce cuál es la relación entre las variables ambientales y la actividad de los murciélagos filostómidos en Honduras. Además, la información disponible en el país en los últimos años sobre este grupo es insuficiente, debido que sólo se conocen registros esporádicos de diversas especies, por ejemplo: Goodwin (1942), Davis et al. (1964), Carter et al. (1966) y LaVal (1969). Estos estudios aportaron los primeros registros de distribución de murciélagos en Honduras. Aunque los registros se mantienen, no se han generado estudios sobre comportamiento, alimentación y ecología con las 113 especies reportadas para el país (Turcios-Casco et al. 2020).

Debido a la escasez de información sobre las condiciones ecológicas en las que se pueden capturar murciélagos filostómidos en Honduras, el objetivo de este estudio fue describir algunos patrones de actividad (hora de captura, temperatura y humedad relativa del aire) de 7 especies de murciélagos filostómidos en Ciudad Universitaria de la Universidad Nacional Autónoma de Honduras (CU-UNAH), Honduras.

La CU-UNAH está ubicada en el municipio del Distrito Central (140 5' 13.63" N y 870 9' 59.72" O; Figura 1), en el Departamento de Francisco Morazán (centro de Honduras), a una elevación promedio de 1,061 m. El suelo se caracteriza por ser arcilloso, la precipitación anual en esta zona es de $869 \mathrm{~mm}$, con una temperatura anual de $22.1^{\circ} \mathrm{C}$ y humedad relativa anual de $77.7 \%$ (Canales 2011); y la evapotranspiración potencial anual es de 1,463 mm 
(DARNA 2013). Sin embargo, al ser un área urbana inmersa en un bosque seco, presenta sitios alterados con predominancia de las siguientes especies vegetales: Lonchocarpus sanctuarii, Acacia farnesiana, Psidium guajava, Muntingia calabura, Solanum erianthum, S. torvum, Tabebuia rosea, Tecoma stans y Spathodea campanulata (Ferrufino et al. 2015).

Realizamos capturas desde el 26 de febrero de 2015 hasta el 29 de abril de 2016, con un total de 21 jornadas. Los sitios se alternaban para no repetir el mismo en la jornada siguiente, y seleccionamos los días de captura de acuerdo con la fase lunar (Erkert 1982; Elangovan y Marimuthu 2001; Ciechanowski et al. 2007) para evitar fobia lunar por parte de los murciélagos, y la presencia de lluvia para que la red no fuese fácilmente detectada (Parsons et al. 2003; Ciechanowski et al. 2007; Voigt et al. 2011). Esto permitió reducir el impacto de dichas variables ambientales sobre la abundancia de las especies. Los únicos meses que no se muestrearon fueron de junio a agosto ya que la universidad permaneció cerrada los días adecuados para la captura de murciélagos de acuerdo con las condiciones anteriormente mencionadas.

Seguimos a Kunz y Kurta (1988) para la posición y colocación de las redes de acuerdo con la vegetación, topografía y cuerpos de agua. Utilizamos dos redes de niebla (14 x $2.5 \mathrm{~m}$, con ojo de malla de $22 \mathrm{~mm}$ ) que permanecieron abiertas desde las 16:30 hr hasta las 00:30 hr para abarcar diferentes ámbitos temporales de acuerdo con los gremios tróficos, y se revisaron cada 20 minutos para disminuir la probabilidad de muerte de los individuos o el escape de la red. Seguimos a Bracamonte (2010) en el tiempo de apertura de las redes al comenzar la actividad de los murciélagos y permanecieron así por 6 horas, aprovechando el período de mayor actividad. El esfuerzo de muestreo en horas/red por metro de red se calculó según Medellín (1993). Para la toma de medidas y la identificación de individuos seguimos a Timm et al. (1999)y Medellín et al. (2008). Todos los individuos fueron manipulados según las pautas de la American Society of Mammalogist para el uso de mamíferos silvestres (Sikes et al. 2016).

Realizamos una prueba de ANOVA de una sola vía con el programa estadístico R 3.4.2 (R Core Team 2015) para determinar la media de los datos de temperatura y la humedad relativa del aire de las horas de inicio y finalización durante cada jornada de los informes del 2015 y 2016 del higrotermográfo de la Estación Meteorológica Experimental (EME) de la UNAH. Ésta se encuentra a una distancia entre $247-$ $732 \mathrm{~m}$ de los sitios seleccionados.

Se capturaron 114 individuos (0.08 individuos/horas/ metro/red) en 1,428.24 horas/metro/red, pertenecientes a 7 especies ( 0.005 especies/hora/metro/red) en 21 jornadas de trabajo. Según el análisis ANOVA, la media de la temperatura de las horas de capturas de los murciélagos filostómidos fue entre $17.4^{\circ} \mathrm{C}$ y $22.8^{\circ} \mathrm{C}$, y la humedad relativa del aire fue entre $58 \%$ y $93.5 \%$ (Figura 2).

La mayor actividad de murciélagos se registró entre las 18:00 - 21:59 hr, en el cual se capturó el $84 \%$ de los individuos, y el otro $16 \%$ fue capturado entre 17:00 - 17:59 hr y 22:00 -

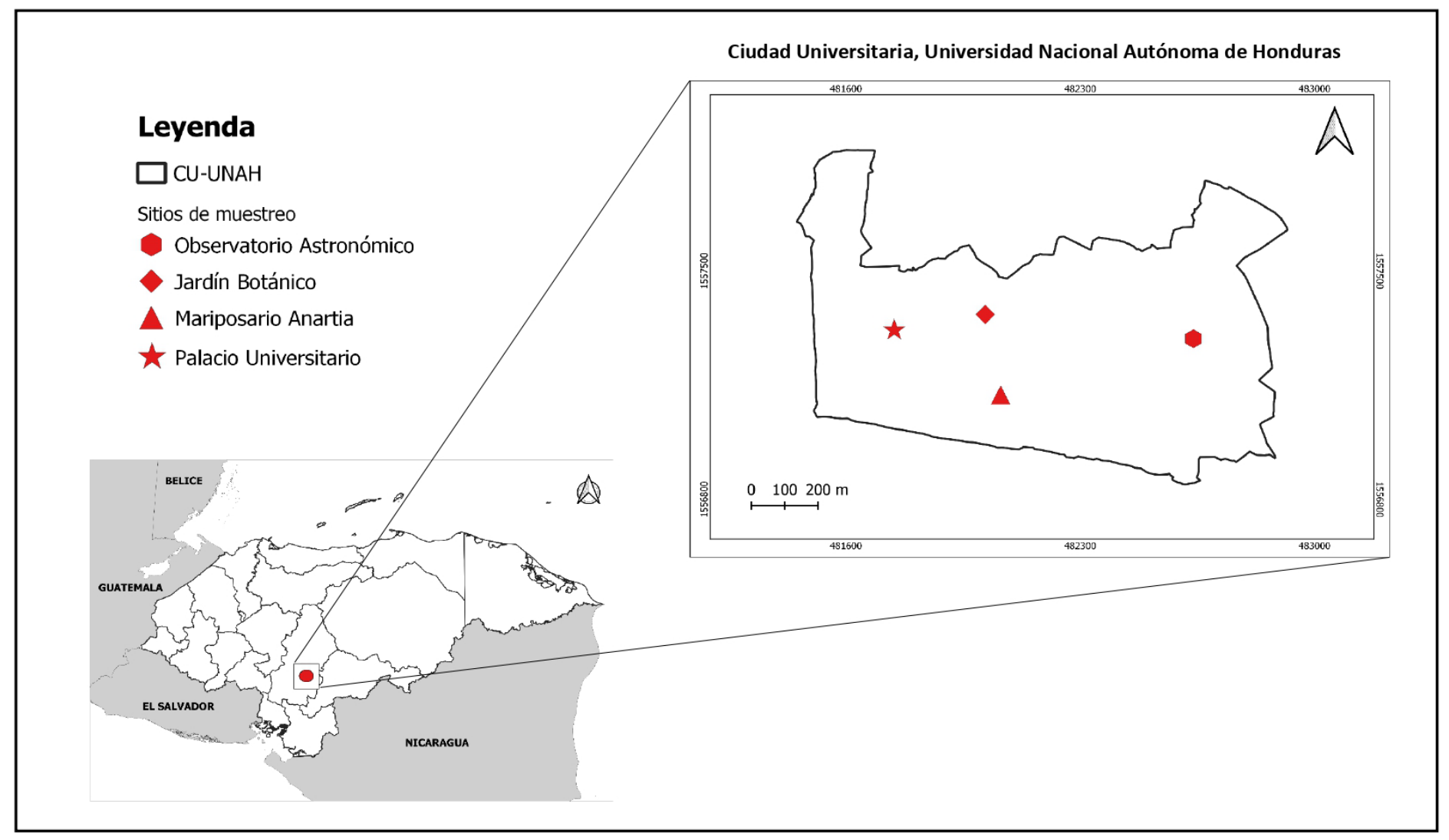

Figura 1. Mapa de los 4 sitios de muestreo en Ciudad Universitaria de la Universidad Nacional Autónoma de Honduras (CU-UNAH) en el Departamento de Francisco Morazán. Alred-

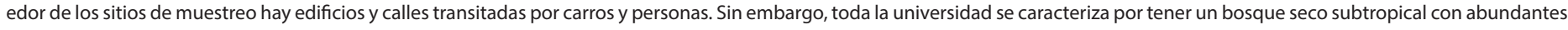
áreas alteradas, y ciertas zonas de bosques en donde se encuentra la mayor cantidad de árboles frutales (por ejemplo, el Jardín Botánico representado por un rombo). 

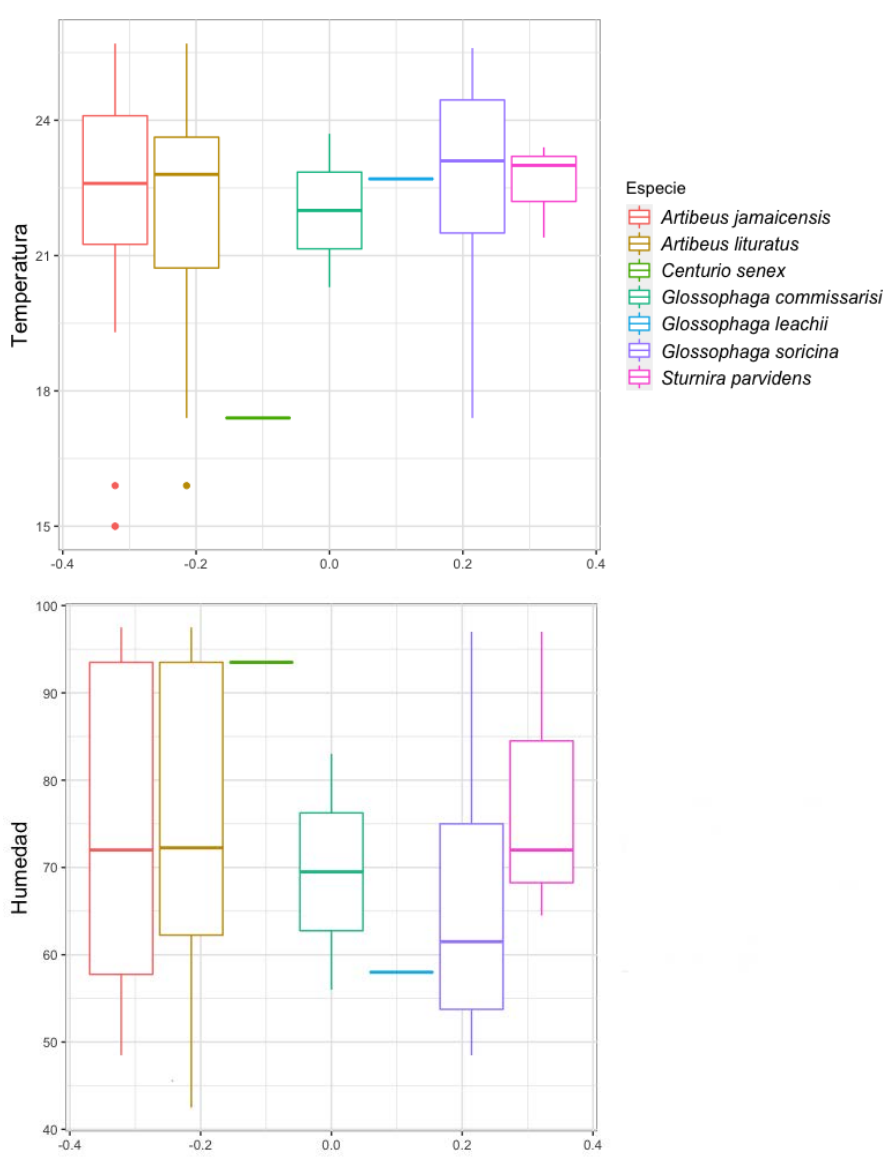

Figura 2. Medias de la humedad relativa (arriba) y temperatura del aire (abajo) en las que se capturaron los murciélagos filóstomidos en Ciudad Universitaria de la Universidad Nacional Autónoma de Honduras (CU-UNAH) en el Departamento de Francisco Morazán en el centro de Honduras. Las líneas horizontales representan la media de las temepraturas; las líneas horizontales superiores e inferiores son los quartiles; y las líneas verticales inferiores y superiores son los rangos respectivos. Los puntos son valores atípicos con respecto a la media.

00:59 hr. Las horas de captura variaron para cada especie; por ejemplo, el $86 \%$ de los individuos $(n=47)$ de A. jamaicensis y el $89 \%$ de los individuos $(n=34)$ de $A$. lituratus se capturaron entre las 17:00 y 20:59 hr. Y el $50 \%$ de las capturas de $G$. soricina se realizaron entre 19:00 y 20:49 hr. Los rangos de las otras especies de murciélagos filostómidos se describen en la Tabla 1. El $22.5 \%$ de las capturas se realizó en febrero, $11.7 \%$ en marzo, $9 \%$ en abril, $12.6 \%$ en octubre, $32.43 \%$ en noviembre y $11.7 \%$ en diciembre.
Artibeus jamaicensis, A. lituratus y Glossophaga soricina fueron las especies más comunes y dos especies fueron representadas por solamente una captura: G. leachii y Centurio senex. Artibeus jamaicensis fue la especie que se capturó en un ámbito temporal más amplio, y $C$. senex la que se capturó a la temperatura más baja y a la humedad relativa más alta. Sin embargo, G. soricina fue la especie que se capturó en la temperatura más alta y $G$. leachii en la humedad relativa más baja.

A diferencia de nuestros resultados, Amorim et al. (2012) encontraron correlaciones estadísticamente significativas entre la actividad de murciélagos de la familia Vespertilionidae con la temperatura y la humedad relativa del ambiente. Se debe considerar que la subfamilia Stenodermatinae (Artibeus, Centurio, Sturnira) y Glossophaginae (Glossophaga) contiene especies generalistas (Reid 2009), por lo que invierten menos tiempo en la búsqueda de alimento (Townsend et al. 2008), a diferencia de especies especialistas como la familia Vespertilionidae, que tienen horarios específicos (vespertinos) para la búsqueda de alimento. La actividad de los murciélagos depende del tiempo en que los murciélagos buscan su alimento, refugio, agua, entre otros recursos, por lo que el tiempo invertido en la búsqueda de alimentos podría estar influenciado por las variables climáticas en algunas especies de murciélagos (Filho et al. 2010; Vela-Vargas y Pérez-Torres 2012). Greenhall (1976) menciona que las humedades relativas del aire entre 55 y $95 \%$ y temperaturas ambientales entre 21.1 y $29.4{ }^{\circ} \mathrm{C}$ son adecuadas para mantener $A$. jamaicensis en cautiverio. Suárez-Payares y Lizcano (2011) registraron un promedio de humedad relativa de $73.7 \%$ y una temperatura ambiental de $24.6^{\circ} \mathrm{C}$ en refugios de $A$. jamaicensis. En México, $G$. leachii se ha capturado en lugares donde las temperaturas mensuales promedio oscilan entre 23.8 y $25.8{ }^{\circ} \mathrm{C}$ (GarcíaGarcía y Santos-Moreno 2014). Este estudio es el primero en describir los patrones de actividad en CU-UNAH. El único estudio que se ha llevado a cabo sobre murciélagos en CU-UNAH fue la descripción de la dieta de murciélagos filóstomidos porTurcios-Casco et al. (2019). Las especies del género Artibeus, consideradas por Emmons y Feer (1999) como especies típicas de áreas perturbadas, resultaron ser las más abundantes representando el $79.8 \%$. Las especies

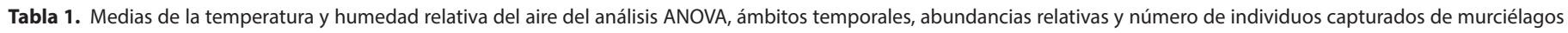
filostómidos en Ciudad Universitaria de la Universidad Nacional Autónoma de Honduras (CU-UNAH) en el Departamento de Francisco Morazán en el centro de Honduras.

\begin{tabular}{|c|c|c|c|c|c|c|c|}
\hline Especie & $\begin{array}{c}\text { Ámbito } \\
\text { temporal (h) }\end{array}$ & $\begin{array}{c}\text { Media de la } \\
\text { temperatura del } \\
\text { aire }\left({ }^{\circ} \mathrm{C}\right)\end{array}$ & $\begin{array}{l}\text { Desviación } \\
\text { estándar }\end{array}$ & $\begin{array}{l}\text { Media de la humedad } \\
\text { relativa del aire (\%) }\end{array}$ & $\begin{array}{l}\text { Desviación } \\
\text { estándar }\end{array}$ & $\begin{array}{l}\text { Abundancia } \\
\text { relativa }\end{array}$ & $\begin{array}{c}\text { Abundancia } \\
\text { total }\end{array}$ \\
\hline Artibeus jamaicensis & $17: 36-00: 10$ & 22.35 & \pm 2.38 & 74.74 & \pm 17.24 & 0.48 & 55 \\
\hline Artibeus lituratus & $17: 50-22: 25$ & 22.23 & \pm 2.44 & 75.02 & \pm 17.45 & 0.32 & 36 \\
\hline Centurio senex & $20: 28$ & 17.4 & 0 & 93.5 & 0 & 0.01 & 1 \\
\hline Glossophaga commissarisi & $19: 22-21: 56$ & 22 & \pm 2.40 & 69.5 & \pm 19.09 & 0.02 & 2 \\
\hline Glossophaga leachii & $18: 56$ & 22.7 & 0 & 58 & 0 & 0.01 & 1 \\
\hline Glossophaga soricina & $18: 40-23: 14$ & 22.83 & \pm 2.31 & 66.16 & \pm 16.95 & 0.14 & 16 \\
\hline Sturnira parvidens & $21: 14-22: 14$ & 21.47 & \pm 1.13 & 75.33 & \pm 22.98 & 0.03 & 3 \\
\hline
\end{tabular}


de Artibeus son consideradas importantes para la regeneración del paisaje debido a que son dispersores de semillas en bosques tropicales (Flores-Martínez et al. 1999). Además de las variables estudiadas, la actividad de los murciélagos puede estar influenciada por la altitud (Linden et al. 2014), tipo de bosque (Owen et al. 2004), o actividad antropológica (Gehrt y Chelsvig 2003; Berthinussen y Altringham 2012). Los resultados de este trabajo demuestran que podrían haber variaciones en los patrones de actividad en las siete especies de murciélagos filostómidos. Recomendamos estudiar la actividad de murciélagos filóstomidos con relación a otros factores ecológicos (e.g., fenofases en las que se encuentran las plantas, cuerpos de agua más cercanos), considerando variaciones estacionales, e incluyendo otros aspectos de comportamiento (e.g., selección de refugios) y ecológicos (e.g., variación en la dieta).

\section{Agradecimientos}

Agradecemos al personal del laboratorio de Zoología de Vertebrados de la Escuela de Biología; al Herbario TEFH; al personal de la Estación Meteorológica Experimental (EME) de la CU-UNAH. A los estudiantes de la Escuela de Biología que participaron a lo largo de toda la investigación, especialmente a C. Antúnez, C. Mejía, V. Henríquez y J. Zúniga. Al personal del Departamento de Vida Silvestre del Instituto Nacional de Conservación y Desarrollo Forestal, Áreas Protegidas y Vida Silvestre (ICF) por el permiso de investigación y de colecta otorgado (Resolución-DE-MP-64-2017). Al personal de la colección de Zoología de la Escuela Agrícola Panamericana (EAP). A M. Canales, N. Estrada, L. Ferrufino, J. Orozco y a cinco revisores anónimos que aportaron valiosos comentarios a este documento.

\section{Literatura citada}

Amorim, F., H. Rebelo, y L. Rodrigues. 2012. Factors influencing bat activity and mortality at a wind farm in a Mediterranean region. Acta Chiropterologica 14:439-457.

ARITA, H. T. 1990. Noseleaf morphology and ecological correlates in phyllostomid bats. Journal of Mammalogy 71:36-47.

Arita, H. T., y M. B. Fenton. 1997. Flight and echolocation in the ecology and evolution of bats. Trends in Ecology \& Evolution 12:53-58.

Berthinussen, A., y J. Altringham. 2012. The effect of a major road on bat activity and diversity. Journal of Applied Ecology 49:82-89.

Bracamonte, J. C. 2010. Murciélagos de bosque montano del Parque Provincial Potrero de Yala, Jujuy, Argentina. Mastozoología Neotropical 17:361-366.

Canales, C. 2011. Cálculo de umbrales para la ciudad de Tegucigalpa. Producto 1 del Proyecto Fondo de Adaptación: "Enfrentando riesgos climáticos en recursos hídricos en Honduras: Incrementando resiliencia y disminuyendo vulnerabilidades en áreas urbanas pobres". Universidad Nacional Autónoma de Honduras, Instituto Hondureño de Ciencias de la Tierra. Tegucigalpa, Honduras.

Carter, D. C., R. H. Pine, y W. B. Davis. 1966. Notes on Middle American bats. The Southwestern Naturalist 11:488-499.
Ciechanowski, M., T. ZająC, A. BıŁAS, y R. Dunajskı. 2007. Spatiotemporal variation in activity of bat species differing in hunting tactics: effects of weather, moonlight, food abundance, and structural clutter. Canadian Journal of Zoology 85:12491263.

Cirranello, A., N. B. Simmons, S. Solari, y R. J. Baker. 2016. Morphological diagnoses of higher-level phyllostomid taxa (Chiroptera: Phyllostomidae). Acta Chiropterologica 18:39-71.

Davis, W. B., F. C. Carter, y R. H. Pine. 1964. Noteworthy records of Mexican and Central American bats. Journal of Mammalogy 45:375-387.

Departamento de Auditoría Sector Recursos Naturales y Ambiente (DARNA). 2013. Auditoría coordinada de gestión ambiental al recurso hídrico practicada al Instituto de Conservación y Desarrollo Forestal, Áreas Protegidas y Vida Silvestre (ICF) (Informe número 017-2013-DARNA-RH-ICF-A). Tegucigalpa, Honduras.

Elangovan, V., y G. Marimuthu. 2001. Effect of moonlight on the foraging behaviour of a megachiropteran bat Cynopterus sphinx. Journal of Zoology 253:347-350.

Emmons, L., y F. Feer. 1999. Neotropical rainforest mammals, a field guide. The University of Chicago Press. Chicago, EE.UU.

ERKeRt, H. G. 1982. Ecological aspects of bat activity rhythms. Pp. 201-242 in Ecology of bats (Kunz, T. H., ed.). Springer. New York, EE.UU.

Estrada-Villegas, S., J. Pérez-Torres, y P. Stevenson. 2007. Dispersión de semillas por murciélagos en un borde de bosque montano. Ecotropicos 20:1-14.

Ferrufino, L., O. Oyuela, G. Sandoval, y F. Beltran. 2015. Flora de la ciudad universitaria, UNAH: un proyecto de ciencia ciudadana realizado por estudiantes universitarios. Revista Ciencia y Tecnología 17:112-131.

Filho, O., N. R. Reis, y C. V. Minte-Vera. 2010. Time and seasonal patterns of activity of phyllostomid in fragments of a stational semidecidual forest from the Upper Paraná River, southern Brazil. Brazilian Journal of Biology 70:937-945.

Flores-Martínez, J. J, J. Ortega, y G. Ibarra-Manríquez. 1999. El hábito alimentario del murcíelago zapotero (Artibeus jamaicensis) en Yucatán. Revista Mexicana de Mastozoología 4:22-39.

Galindo-González, J. 1998. Dispersión de semillas por murciélagos: su importancia en la conservación y regeneración del bosque tropical. Acta Zoológica Mexicana (nueva serie) 73:57-74.

García-García, J. L., y A. Santos-Moreno. 2014. Variación estacional en la diversidad y composición de ensambles de murciélagos filostómidos en bosques continuos y fragmentados en Los Chimalapas, Oaxaca, México. Revista Mexicana de Biodiversidad 85:228-241.

Gehrt, S. D., y J. E. Chelsvig. 2003. Bat activity in an urban landscape: patterns at the landscape and microhabitat scale. Ecological Applications 13:939-950.

Goodwin, G. G. 1942. Mammals of Honduras. Bulletin of the American Museum of Natural History 79:107-195.

Greenhall, A. M. 1976. Care in captivity. Pp. 89-126 in Biology of bats of the new world family Phyllostomidae (Baker, R. J., J. K. Jones, Jr., y D. C. Carter, eds.). Texas Tech University Press. Lubbock, EE.UU.

Kalko, E. K., y M. A. Condon. 1998. Echolocation, olfaction and fruit display: how bats find fruit of flagellichorous cucurbits. Functional Ecology 12:364-372. 
Kunz, T. H., Y A. KuRTA. 1988. Capture methods and holding devices. Pp. 1-28 in Ecological and behavioral methods for the study of bats (Kunz, T. H., ed.). Smithsonian Institution Press. Washington, EE.UU.

LAVAL, R. K. 1969. Records of bats from Honduras and El Salvador. Journal of Mammalogy 50:819-822.

Linden, V. M., S. M. Weier, I. Gaigher, H. J. Kuipers, M. J. WeterINGS, Y P. J. TAYLOR. 2014. Changes of bat activity, species richness, diversity and community composition over an altitudinal gradient in the Soutpansberg range, South Africa. Acta Chiropterologica 16:27-40.

Medelíin, R. A. 1993. Estructura y diversidad de una comunidad de murciélagos en el trópico húmedo mexicano. Pp. 333-350 in Avances en el estudio de mamíferos de México (Medellín, R. A., y G. Ceballos, eds.). Asociación Mexicana de Mastozoología. Ciudad de México, México.

Medellín, R. A., H. T. Arita, y O. Sánchez. 2008. Identificación de los murciélagos de México, clave de campo. $2^{\text {a }}$ edición. Universidad Nacional Autónoma de México. Ciudad de México, México.

Muñoz-Romo, M. y E. A. Herrera. 2010. Observaciones sobre la alimentación del murciélago frugívoro mayor Artibeus lituratus (Chiroptera: Phyllostomidae) en Venezuela. Revista Mexicana de Mastozoología 14:51-58.

Neuweiler, G. 1989. Foraging ecology and audition in echolocating bats. Trends in Ecology \& Evolution 4:160-166.

Novoa, S., R. Cadenillas, y V. Pacheco. 2011. Dispersión de semillas por murciélagos frugívoros en bosques del Parque Nacional Cerros de Amotape, Tumbes, Perú. Mastozoología Neotropical 18:81-93.

Orozco-Lugo, L., A. Guillén-Servent, D. Valenzuela-Galván, y H. T. ARItA. 2013. Descripción de los pulsos de ecolocalización de once especies de murciélagos insectívoros aéreos de una selva baja caducifolia en Morelos, México. Therya 4:33-46.

Owen, S. F., M. A. Menzel, y J. W. Edwards. 2004. Bat activity in harvested and intact forest stands in the Allegheny Mountains. Northern Journal of Applied Forestry 21:154-159.

Parsons, K. N., G. Jones, y F. Greenaway. 2003. Swarming activity of temperate zone microchiropteran bats: effects of season, time of night and weather conditions. Journal of Zoology 261:257-264.

R Core Team. 2015. R: A language and Environment for statistical computing. R Foundation for Statistical Computing. Vienna, Austria.

REID, F. A. 2009. A field guide to the mammals of Central America \& southeast Mexico. $2^{\text {nd }}$ edition. Oxford University Press. New York, EE.UU.

Sikes, R. S., y the Animal Care and Use Committee of the American Society of Mammalogists. 2016. 2016 Guidelines of the American Society of Mammalogists for the use of wild mammals in research and education. Journal of Mammalogy 97:663-688.

Suárez-Payares, L. M., Y D. J. Lizcano. 2011. Uso de refugios por tres especies de murciélagos filostómidos (Chiroptera: Phyllostomidae) en el Área Natural Única Los Estoraques, Norte de Santander, Colombia. Mastozoologia Neotropical 18:259-270.

Tımm, R. M., R. K. LAVAL, Y B. Rodríguez-H. 1999. Clave de campo para los murciélagos de Costa Rica. Brenesia 52:1-32.

Townsend, C. R., M. Begon, y J. L. Harper. 2008. Essentials of ecology. $3^{\text {rd }}$ edition. Blackwell Publishing. Victoria, Australia.
Turcios-Casco, M. A., H. D. Ávila-Palma, E. J. Ordoñez-Trejo, A. Soler-Orellana, y D. I. Ordoñez-Mazier. 2019. Comments on the diet of phyllostomid bats (Chiroptera) in a subtropical dry forest in central Honduras. Studies on Neotropical Fauna and Environment 54:3:239-244.

Turcios-Casco, M. A., H. D. Ávila-Palma, R. K. LaVAL, R. D. Stevens, E. J. Ordoñez-Trejo, J. A. Soler-Orellana, y D. I. OrdoñezMAzIer. 2020. A systematic revision of the bats (Chiroptera) of Honduras: an updated checklist with corroboration of historical specimens and new records. Zoosystematics and Evolution 96:411-429.

Vargas Espinoza, A., L. F. Aguirre, M. I. Galarza, y E. Gareca. 2008. Ensamble de murciélagos en sitios con diferente grado de perturbación en un bosque montano del Parque Nacional Carrasco, Bolivia. Mastozoología Neotropical 15:297-308.

Vela-Vargas, I. M., y J. Pérez-Torres. 2012. Murciélagos asociados a remanentes de bosque seco tropical en un sistema de ganadería extensiva (Colombia). Chiroptera Neotropical 18:1089-1100.

Voigt, C. C., K. Schneeberger, S. L. Voigt-Heucke, y D. Lewanzik. 2011. Rain increases the energy cost of bat flight. Biology Letters 7:793-795.

Editor asociado: José F. Moreira-Ramírez Sometido: Noviembre 17, 2020; Revisado: Febrero 16, 2021. Aceptado: Febrero 24, 2021; Publicado en línea: Marzo 10, 2021. 